\title{
Monitoring of the Time Change in Transfer Functions in the Central Japan Conductivity Anomaly
}

\author{
M. SHIRAKI \\ Kakioka Magnetic Observatory, Kakioka, Ibaraki-ken, Japan
}

(Received August 16, 1979; Revised May 28, 1980)

\begin{abstract}
Time change in transfer functions for conductivity anomaly in central Japan is being monitored to detect possible electrical conductivity changes preceding earthquakes. Using geomagnetic data with a sampling interval of one minute, the transfer functions at the Kakioka Magnetic Observatory are calculated day by day. The method of analysis is based on the power spectrum analysis as developed by Everett and Hyndman. Monthly means are statistically obtained from these daily values of transfer functions. Monthly transfer functions during 40 months from January 1976 to April 1979 showed no significant changes. Small fluctuations are likely to be accounted for by seasonal changes probably originating in the external magnetic disturbance. No major earthquake activity has been reported in the vicinity of this site during this period.
\end{abstract}

\section{Introduction}

The peculiar behaviour of the vertical component of short-period geomagnetic variation has been observed in many regions of the world. This is now interpreted in terms of the anomalous distribution of conductors in the crust and upper mantle and is called the conductivity anomaly. In a conductivity anomaly region, the vertical component of short-period geomagnetic variation $\Delta Z$ is related to the horizontal components, $\Delta H$ and $\Delta D$, as empirically expressed by the linear equation

$$
\Delta Z=A \Delta H+B \Delta D \text {. }
$$

The coefficients $A$ and $B$ are constants peculiar to a given station. Generally speaking, they are frequency-dependent complex functions and are referred to as transfer functions. The feature of the conductivity anomaly is generally well described by these transfer functions. In Japan, the most important and well investigated anomaly is the central Japan anomaly found by Rikitake and his coworkers (RIKITAKE, 1959). This anomaly is in harmony with a heat flow distribution and has been explained by the undulation of the upper mantle conducting layer (RIKITAKE, 1969).

On the other hand, in a seismically active region, time changes in the underground electrical conductivity are predicted by the dilatancy model (ScHOLz et al., 1973). 
Such changes have been observed by the artificial source DC sounding with special reference to the occurrence of earthquakes (SADovsky et al., 1972; MAZzELLA and MORRISON, 1974). These facts may indicate that the transfer functions are timedependent in earthquake regions. Moreover, Rikitake (1976) showed from model calculations that it is possible to detect time changes in transfer functions in shortperiod geomagnetic variations if the conductivity changes appreciably in association with earthquakes. Indeed, some evidence supporting the time dependence of transfer functions have recently been reported by a few researchers, particularly in Japan (Yanagihara, 1972; MiYakoshi, 1975; Yanagihara and Nagano, 1976; Rikitake, 1979). Their results were reviewed by NiblETt and HonkuRA (1978).

The earliest important study was that of YANAGIHARA (1972), who found quite a remarkable secular change in transfer functions at the Kakioka Magnetic Observatory and suggested that it might be associated with a very large seismic event. YANAGIHARA and NAGANO (1976) further showed quite rapid changes in transfer functions at this observatory over a period of a few weeks before and after some major seismic events. Kakioka is located in the central Japan conductivity anomaly region and lies over a well-known seismically active region. So their results are not only interesting for the elucidation of conductivity anomaly itself, but also very important for the practical application to the future research on earthquake prediction.

Therefore, as a feasible study, the time changes in transfer functions at Kakioka are now being monitored to detect possible electrical conductivity changes preceding earthquakes. The results during the 40 months from January 1976 to April 1979 are reported in this paper, though significant changes, with special reference to earthquake occurrence, have not been observed. This may be because no major earthquake has been reported in this site during this period. However, it is important to show the time dependence of transfer functions during a period without any major seismic activity.

\section{Data Analysis}

At the Kakioka Magnetic Observatory, a new system consisting of optical pumping magnetometers are now in operation (YANAGIHARA et al., 1973), and digital data recorded on magnetic tapes at a sampling interval of one minute have become available for analysis since the beginning of 1976. Using these digital data, time changes in transfer functions are being monitored.

In the present study, transfer functions are obtained by making use of the method of power spectrum analysis. Auto- and cross-power spectra of the data are calculated using the technique of BLACKMAN and TUKEY (1958), after the high-pass filter is digitally operated to the raw data. Calculations are made day by day for 24-hour nonoverlapping data sets of three components. The maximum lag time for the power spectrum estimate is chosen as $60 \mathrm{~min}$. So the range of frequency of estimated power 
spectra is from 1 cycle to 60 cycles per $120 \mathrm{~min}$. Transfer functions are derived from these power spectra according to the formula presented by EVERETT and HYNDMAN (1967):

$$
\begin{array}{r}
A=\frac{P_{Z H} P_{D D}-P_{D H} P_{Z D}}{P_{H H} P_{D D}-P_{H D} P_{D H}} \\
B=\frac{P_{Z D} P_{H H}-P_{H D} P_{Z H}}{P_{H H} P_{D D}-P_{H D} P_{D H}}
\end{array}
$$

where $P_{H H}, P_{D D}$ denote the auto-power spectra and $P_{Z H}$, and the like, the cross-power spectra. These functions are generally complex functions and are written as

$$
\begin{aligned}
& A=A_{u}+i A_{v} \\
& B=B_{u}+i B_{v} .
\end{aligned}
$$

Day-to-day calculation of transfer functions has been carried out during the 40 months from January 1, 1976 to April 30, 1979, regardless of the degree of magnetic disturbance. Such a disposal of data is not the best one for precise analysis, but the simplest one for objective analysis by an automated computer technique. Inadequate
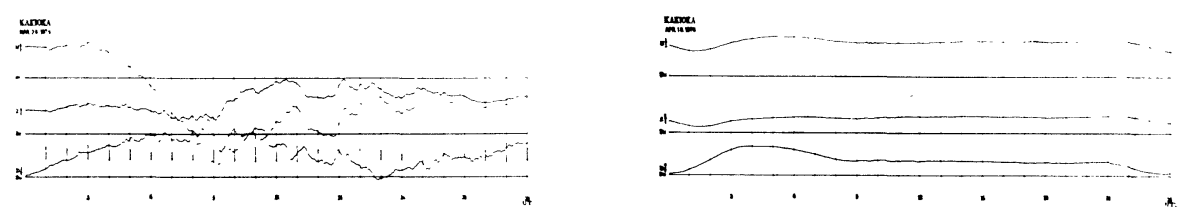

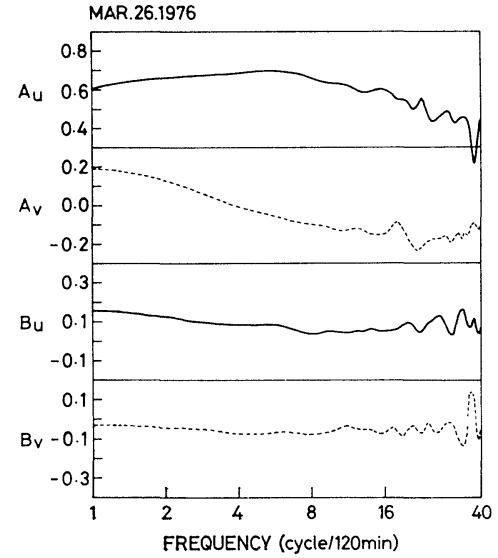

(a)

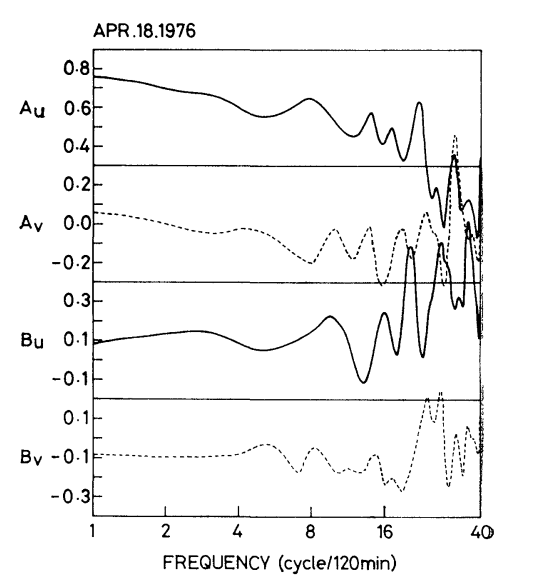

(b)

Fig. 1. Examples of transfer functions at the Kakioka Magnetic Observatory for a geomagnetically disturbed day (a) and a calm day (b). 
points in this disposal are excluded statistically by the secondary disposal of the daily transfer functions in the next section.

Transfer functions for two days are shown in Fig. 1 by way of examples. Figure 1a shows the result for a magnetically highly disturbed day (March 26, 1976), and Fig. 1b shows the result for a calm day (April 18, 1976). The daily sum of $K$ index $(\Sigma K)$ at Kakioka is 41 for the disturbed day, and 2 for the calm day. Magnetograms used for analyses are shown in the upper part of the respective figures.

As clearly seen in Fig. 1, transfer functions change smoothly over the given frequency range for the disturbed day, but they are oscillatory at a high-frequency range for the calm day. These statements generally hold true for other disturbed and calm days, respectively. The oscillatory feature of transfer functions for calm days may be due to a low signal-to-noise ratio for high-frequency variations. However, it is interesting to note that the values of transfer functions at frequencies lower than 8 cycles per $120 \mathrm{~min}$ (periods longer than $15 \mathrm{~min}$ ) for calm days are nearly equal to those for disturbed days. Therefore, time changes of transfer functions are examined in the present study for the 4 periods of $15,20,30$, and $60 \mathrm{~min}$. However, the results are shown only for the period of $30 \mathrm{~min}$ because those for the other periods are nearly similar to the former.

Figure 2 shows daily values of transfer functions for a period of 30 min during the

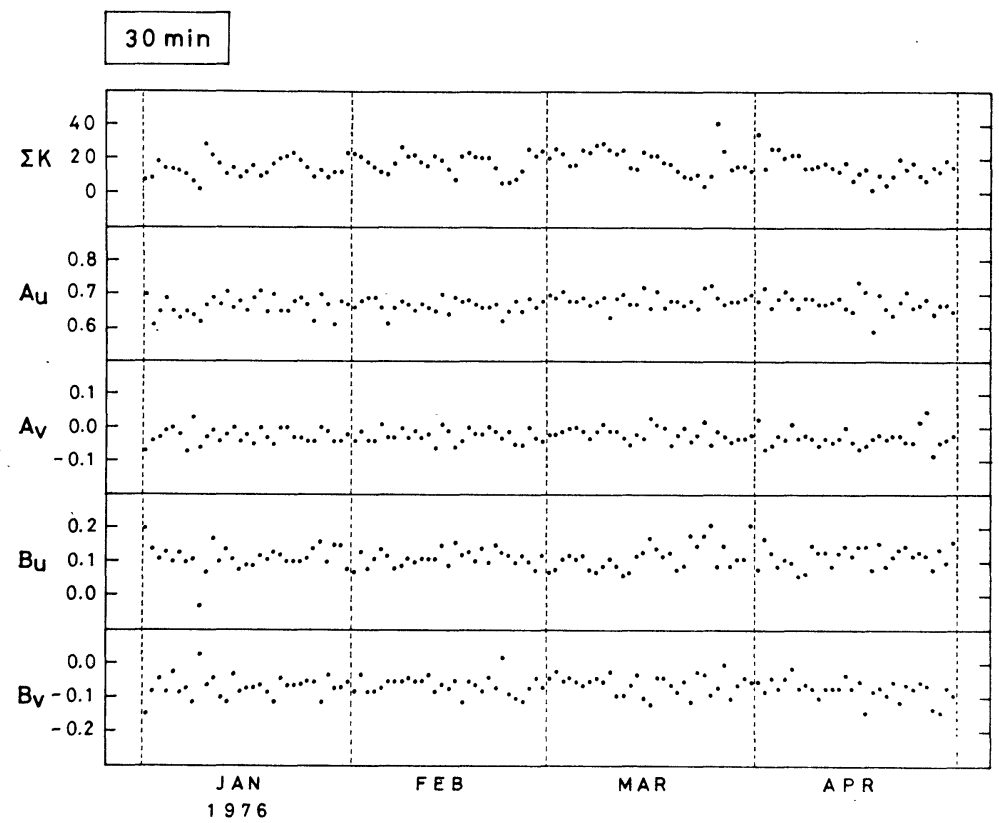

Fig. 2. Day-to-day changes of transfer functions at the Kakioka Magnetic Observatory during the first 4 months of 1976. 
first 4 months of the present analysis. The variance of the day-to-day change is the smallest for this period, compared with those for the other three periods. This is the reason why the results for the period of $30 \mathrm{~min}$ are shown as the representative ones. However, maximum scatters in the figure are $0.15,0.13,0.24$, and 0.17 for $A_{u}, A_{v}, B_{u}$, and $B_{v}$, respectively. Such day-to-day changes of transfer functions cannot be ascribed to changes of electrical conductivity in the earth. One of the causes could be attributable to the assumptions made in the transfer function analysis which are not necessarily satisfied in the actual cases, another to low signal-to-noise ratios for many of the calm days.

\section{Monthly Mean Analysis}

If the day-to-day scatter of transfer functions is random, it can be eliminated, to a

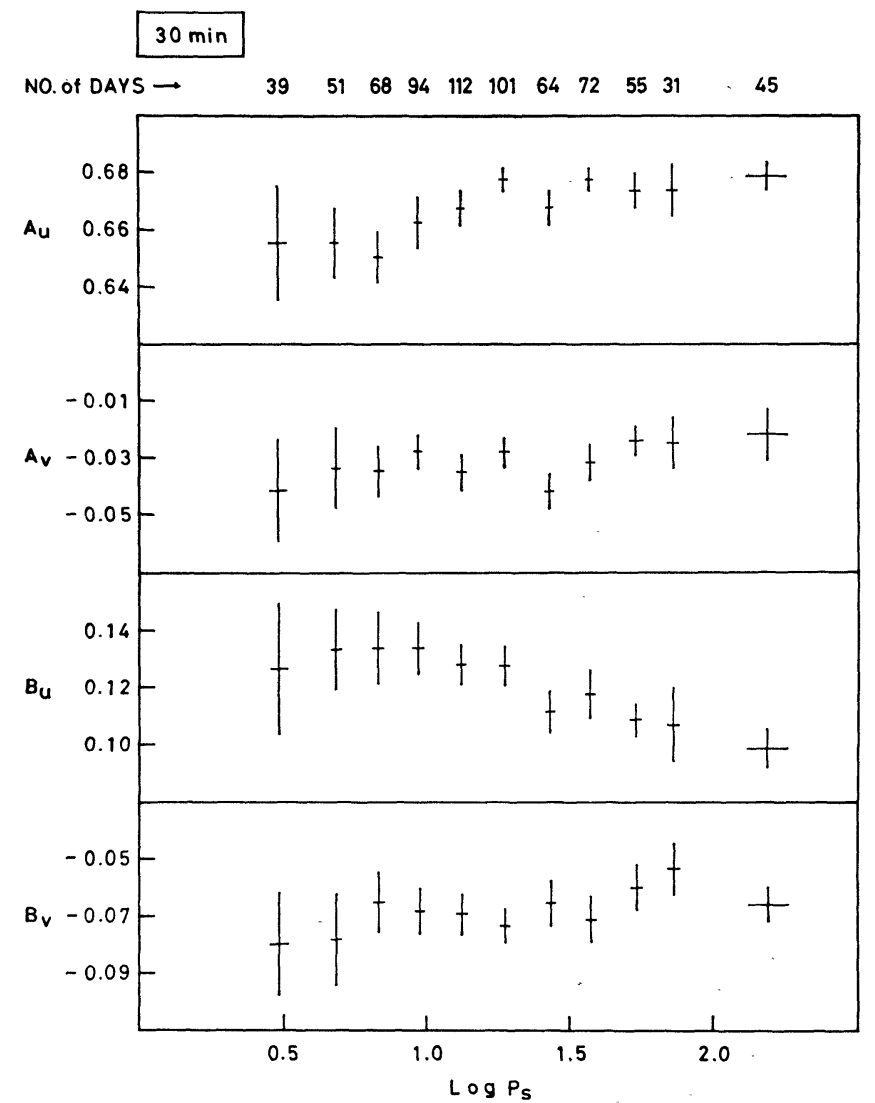

Fig. 3. Correlations between the values of 'transfer functions and the logarithmic values of $P_{s}$. 
certain extent, by taking averages, although the time resolution of changes as a precursor would be reduced as a result. However, it was found from detailed examination that the scatter is not random. The changes of daily transfer functions are somewhat correlated with the degree of magnetic disturbance which can be represented by the daily sum of $K$ index or by the daily power of $H$ or $D$ component.

Figure 3 shows the mean values of the transfer functions determined for 11 groups according to the degree of disturbance. For the degree of disturbance, the logarithmic value of $P_{s}$ is adopted, where $P_{s}$ is the sum of the powers of $H$ and $D$ components. The period considered is $30 \mathrm{~min}$ and the means are obtained from data of the 2 years, 1976 and 1977. Vertical and horizontal lines denote the $95 \%$ confidence intervals of the respective means. The numbers of days for the calculation of the means are given for each group.

As shown in the figure, the scatter of transfer functions tends to become large for

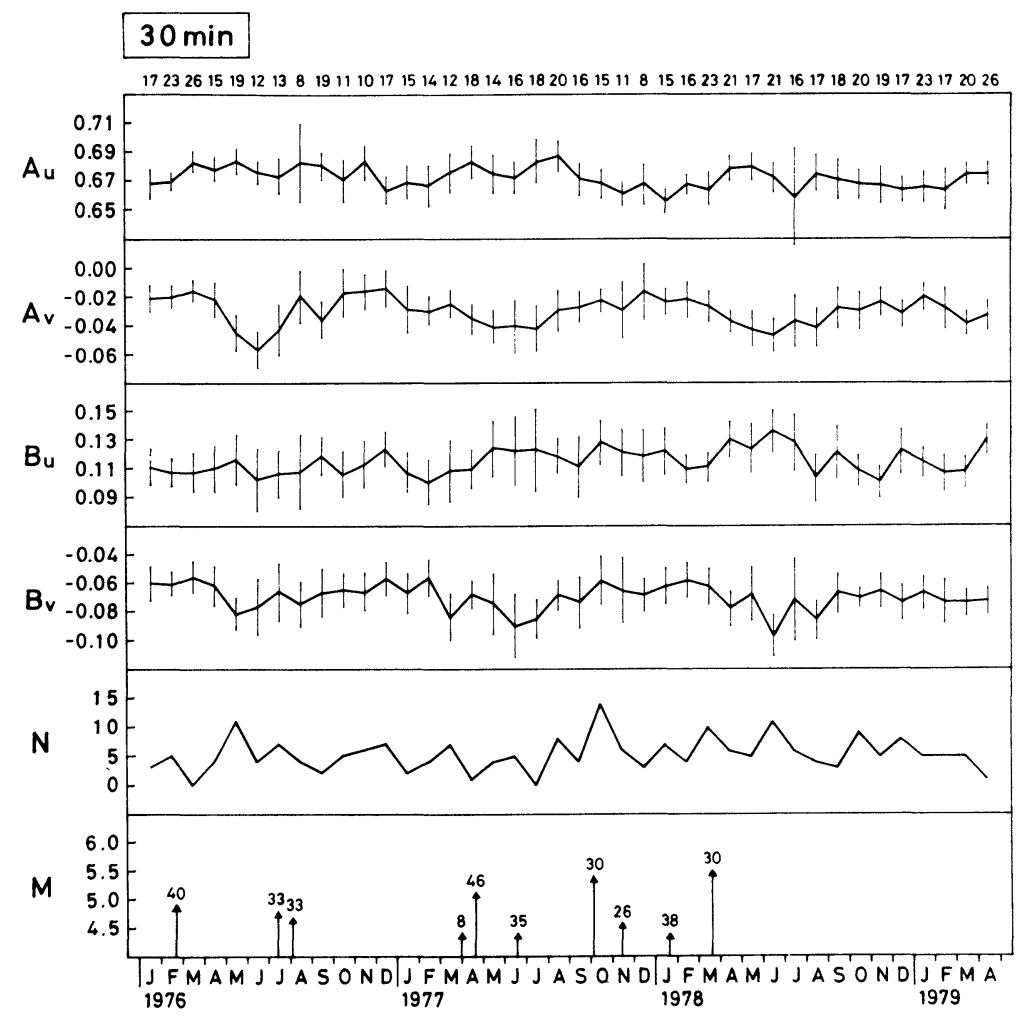

Fig. 4. Monthly changes of transfer functions with a threshold value of $\log P_{s}$ and the number of earthquakes felt at Kakioka. Earthquakes of magnitude greater than 4.3 within a radius of $50 \mathrm{~km}$ of the Kakioka Magnetic Observatory are shown with their approximate epicentral distance in $\mathrm{km}$. 
low values of $\log P_{s}$. Moreover, the values of transfer functions, especially $A_{u}$ and $B_{u}$, depend considerably on $\log P_{s}$. The value of $A_{u}$ increases with increasing value of $\log P_{s}$, while that of $B_{u}$ decreases. Perhaps, the transfer functions at low values of $\log P_{s}$ would not be reliable because of low signal-to-noise ratios. Therefore, the transfer functions at values of $\log P_{s}$ less than a threshold value are not used for the next monthly mean analysis. Considering the results shown in Fig. 3, the threshold value of $\log P_{s}$ for the period of $30 \mathrm{~min}$ should be chosen as 1.2. Because of the application of this threshold value to the analysis, the number of days used in the calculation of monthly means became about one half of the total number of days.

The monthly means of transfer functions for the period of $30 \mathrm{~min}$ are shown in Fig. 4. The number of days used for each mean is given in the upper part of the figure. The scatter in the monthly means is smaller by about one order of magnitude than the daily scatter. Almost all fluctuations in the figure are insignificant, considering the $95 \%$ confidence limit for the mean which is shown by the straight vertical line. However, some changes are significant. One of the significant changes is the decrease of $A_{u}$ appearing from August to November in 1977. Another is the change of $A_{v}$ lasting from March to August in 1976.

\section{Discussions}

It is rather hasty to conclude that the monthly changes in transfer functions in Fig. 4 are due to changes of electrical conductivity in the earth. It is noted in the previous section that the degree of magnetic disturbance explicitly affects the values of the transfer functions. Though this effect is removed, to a certain extent, by the averaging procedure in which a threshold value of $\log P_{s}$ is taken into account, the monthly changes in transfer functions in Fig. 4 may still include this effect. In addition to $\log P_{s}$, some factors characterizing magnetic disturbances seem to be related to the changes in transfer functions, though the group correlation analysis for these factors does not show explicit correlations as clearly as in the case for $\log P_{s}$ in Fig. 3.

Characteristic factors examined besides $\log P_{s}$ are the degree of polarization $(D e g)$ the coherency $(C o h)$, the angle of polarization $(A n g)$, the ellipticity $(E l l)$, and the spectral slope $(m)$ which can be obtained from the auto- and cross-power spectra of $H$ and $D$ components. The factors except $m$ are calculated by making use of the technique usually used in the study of magnetic pulsations (RANKIN and KURTZ, 1970). The spectral slope $m$ is the coefficient in the following relation between $P_{s}$ and $T$,

$$
\log P_{s}=m \log T+C
$$

where $T$ is a period and $C$ is a constant (CAMPBELL, 1976). The value of $m$ is calculated by the least squares method for the period range from $15 \mathrm{~min}$ to $60 \mathrm{~min}$. The monthly means of above factors determined for days having a value of $\log P_{s}$ greater than 1.2 are illustrated in Fig. 5. Together with them, those of transfer functions are also re- 
illustrated in this figure. It should be noted that the time scale in Fig. 5 is shortened by one fifth of that in Fig. 4.

As seen in Fig. 5, there are certain changes in some of the factors which are simi-

\section{$30 \mathrm{~min}$}
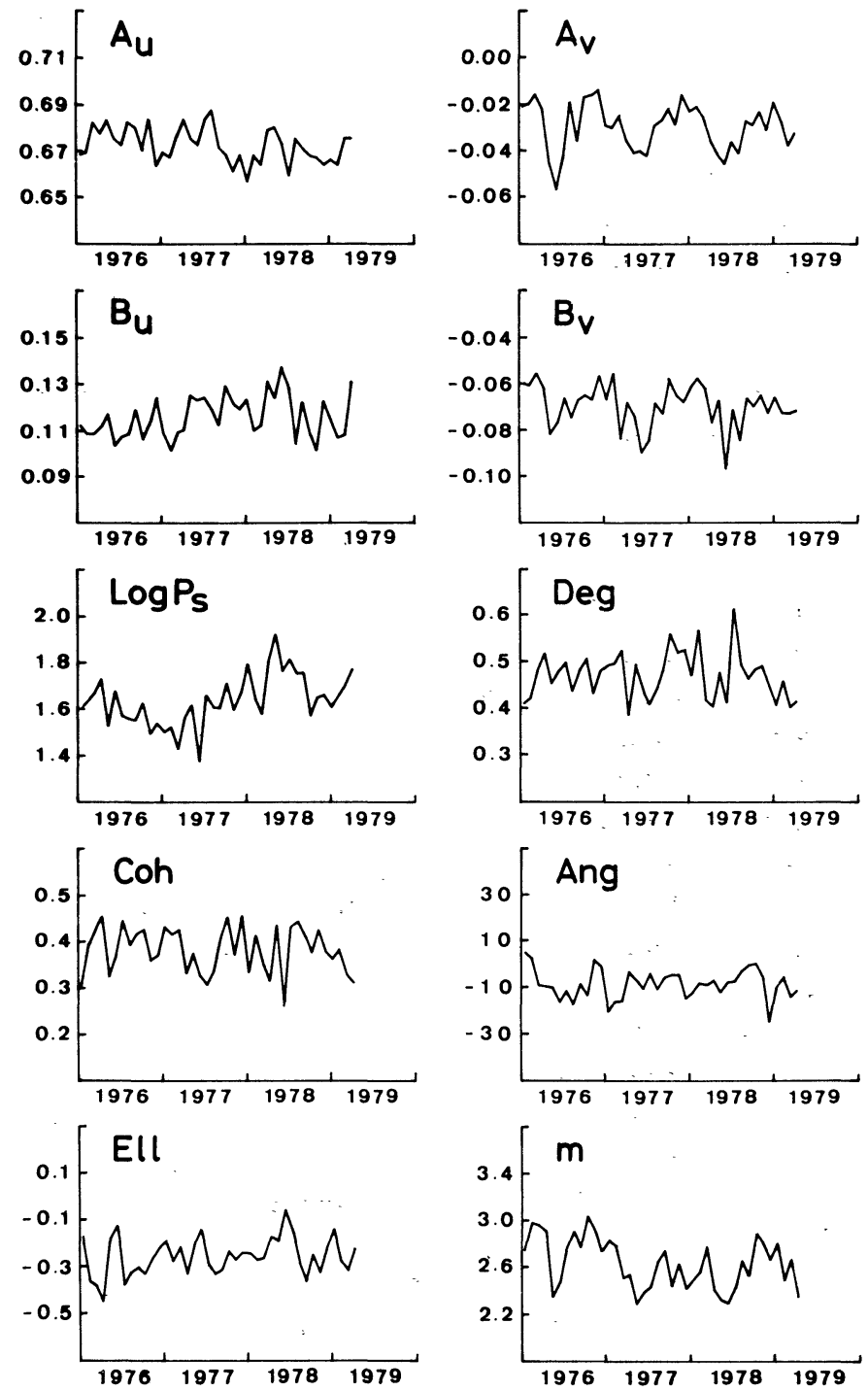

Fig. 5. Monthly changes of transfer functions and characteristic factors of external magnetic disturbance with a threshold value of $\log P_{s}$. 
Table 1. Correlation coefficients between the monthly changes of transfer functions and those of characteristic factors for the external magnetic disturbance. Coefficients underlined are significant at the $95 \%$ confidence limit.

\begin{tabular}{lcrrr} 
& $A_{u}$ & \multicolumn{1}{c}{$A_{v}$} & \multicolumn{1}{c}{$B_{u}$} & \multicolumn{1}{c}{$B_{v}$} \\
Log $P_{s}$ & -0.08 & -0.18 & $\underline{0.37}$ & 0.03 \\
Deg & -0.38 & 0.14 & -0.06 & 0.27 \\
Coh & -0.16 & $\underline{0.31}$ & -0.40 & 0.43 \\
Ang & -0.01 & 0.18 & 0.09 & 0.16 \\
Ell & -0.22 & $\underline{-0.38}$ & $\underline{0.47}$ & -0.46 \\
$m$ & -0.05 & $\underline{0.58}$ & -0.64 & 0.51 \\
\hline
\end{tabular}

lar to those of transfer functions. One example of the similarity is the changes in $A_{u}$ and Deg which appeared from April 1976 to October 1977. Another is the changes in $A_{v}$ and $m$ except during the interval from October 1977 to March 1978. Table 1 gives the correlation coefficients between transfer functions and factors characterizing magnetic disturbances calculated from the results in Fig. 5, and coefficients which are significant at the $95 \%$ confidence level are underlined. Though the correlation is not so high and the mechanism of the correlation is not clear, the results in Fig. 5 and Table 1 may indicate the fact that the changes of transfer functions depend largely on the external magnetic disturbances. Such a fact was also pointed out for the time change of transfer functions in the high latitude zone (HONKURA et al., 1977; ANDERSON et al., 1978).

In examining the changes of transfer functions in Fig. 4 or Fig. 5, it seems that most of their changes can be ascribed to seasonal changes. There is a tendency that

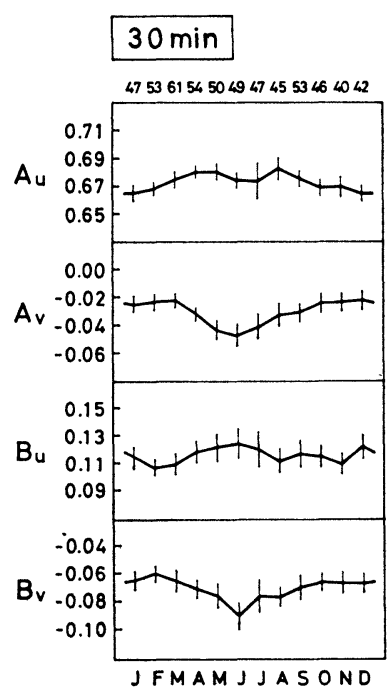

Fig. 6. Mean monthly changes of transfer functions averaged over 3 years from 1976 to 1978. 
$A_{u}$ and $B_{u}$ are small in winter and large in summer, while $A_{v}$ and $B_{v}$ are large in winter and small in summer. This is much clearer in Fig. 6 which shows the mean monthly changes in transfer functions averaged over 3 years from 1976 to 1978 . The existence of such seasonal changes may also indicate that the changes in transfer functions originate in the external magnetic disturbance which undergoes a well-known seasonal change. Therefore, it is not clearly evident that a relation exists between the changes in transfer functions in Fig. 4 and the change of electrical conductivity in the earth.

Taking the above mentioned facts into consideration, the seismic activity near Kakioka is compared with the time changes in transfer functions. As an indication of the seismic activity, the monthly change in the number of earthquakes felt at Kakioka $(N)$ is shown in Fig. 4. In addition, earthquakes of a magnitude $(M)$ greater than 4.3 that occurred within a radius of $50 \mathrm{~km}$ from the Kakioka Magnetic Observatory are indicated in the bottom of Fig. 4 by the vertical arrows with their approximate epicentral distance in $\mathrm{km}$. The data have been reported by the Japan Meteorological Agency and are available at present to the end of October 1978. In Fig. 4, however, no significant correlation is found between the changes in transfer functions and those in seismic activity, partly because no major earthquake which is accompanied by an underground conductivity change has occurred during this period near Kakioka. Even if the earthquakes indicated in the figure affected the values of transfer functions, their effect might be less than their seasonal changes and be concealed by them.

It is clear from Fig. 5 that the range of fluctuations in transfer functions which is the error level for the primary purpose of the present study is about 0.04 at the maximum. On the other hand, the magnitude of the change with special reference to earthquakes has hitherto been reported to be about 0.1 (YanAgIHARA, 1972; MiYaKoshi, 1975; YANAgihara and Nagano, 1976; Rikitake, 1979). Therefore, the changes to be expected in association with earthquakes are well above the error level, and can be significantly detected in the future.

In the present analysis, the resolution of the time change in transfer functions is a month or more because of the disposal of monthly average. Considering the number of days and confidence intervals for the monthly means, it seems unsuitable to calculate the means for an interval less than a month. Therefore, it is difficult to detect such precursory changes having a duration of a few weeks as the ones reported by YANAGIHARA and NAGANO (1976). However, the changes over a few years as reported by MiYAKOSHI (1975) and RIKITAKE (1979) or the secular changes over several decades as reported by YANAGIHARA (1972) can be easily detected from the present monthly analysis.

In regard to such long-term changes, it is interesting to note that the mean levels of the fluctuations of transfer functions seem to increase or decrease gradually over the interval of the present analysis, though the total long-term change itself does not exceed the confidence limit. Fitting the linear equation to the monthly changes, the rate of change is calculated to be -0.0026 per year for $A_{u},-0.0011$ per year for $A_{v}$, 
+0.0028 per year for $B_{u}$, and -0.0021 per year for $B_{v}$. The polarity and amount of the change in $A_{u}$ is consistent with the result reported by YANAGIHARA (1972). One of the possibilities for this long-term trend may be the secular change of electrical conductivity in the earth associated with a future earthquake occurrence. Another possibility is the change originating in the external magnetic disturbance which shows the 11-year variation in accordance with the change in solar activity. Further monitoring is needed in order to obtain a more definite conclusion.

The author would like to express his hearty thanks to Dr. M. Kawamura, the Director of the Kakioka Magnetic Observatory, for his continuous support during this work. The author is very grateful to Dr. K. Yanagihara, the former Director of the Kakioka Magnetic Observatory, for his interest and advice throughout this study. The author thanks Dr. Y. Honkura of Tokyo Institute of Technology for his useful comments on the manuscripts. Helpful discussions with Mr. Y. Sano are gratefully acknowledged. Almost all computation of this work was carried out at the computer center of Japan Meteorological Agency and the help given by the staff of the center is also gratefully acknowledged.

\section{REFERENCES}

Anderson, C. W., L. J. LANzerotti, and C. G. Maclennan, Local time variation of geomagnetic induction vectors, J. Geophys. Res., 83, 3469-3484, 1978.

Blackman, R. B. and J. W. Tukey, The Measurement of Power Spectra, 190pp., Dover Publications, New York, 1958.

CAMPBeLl, W. H., Spatial distribution of the geomagnetic spectral composition for disturbed days, J. Geomag. Geoelectr., 28, 481-496, 1976.

EVERETT, J. E. and R. D. HyNDMAN, Geomagnetic variations and electrical conductivity structure in southern Australia, Phys. Earth Planet. Inter., 1, 24-34, 1967.

HonkuRA, Y., R. D. KuRTZ, and E. R. Niblett, Geomagnetic depth sounding and magnetotelluric results from a seismically active region northeast of Quebec City, Can. J. Earth Sci., 14, 256267, 1977.

Mazzella, A. and H. F. Morrison, Electrical resistivity variations associated with earthquakes on the San Andreas fault, Science, 185, 855-857, 1974.

MiYAKOSHI, J., Secular variation of Parkinson vectors in a seismically active region of Middle Asia, J. Fac. General Education, Tottori Univ., 8, 209-218, 1975.

NibletT, E. R. and Y. HonkURA, Time-dependence of electromagnetic transfer functions and their association with tectonic activity, a review paper presented at the Fourth Workshop on Electromagnetic Induction in the Asthenosphere, in Murnau, September 1978.

RANKIn, D. and R. KuRTz, Statistical study of micropulsations, J. Geophys. Res., 75, 5444-5458, 1970.

RIKITAKE, T., The anomalous behaviour of geomagnetic variations of short period in Japan and its relation to the subterranean structure, The 9th report, Bull. Earthq. Res. Inst., 37, 545-570, 1959.

RIKITAKE, T., The undulation of an electrically conducting layer beneath the island of Japan, Tectonophysics, 7, 257-264, 1969.

RIKITAKE, T., Crustal dilatancy and geomagnetic variations of short period, J. Geomag. Geoelectr., 28, 145-156, 1976.

RIKITAKE, T., Changes in the direction of magnetic vector of short-period geomagnetic variations before the 1972 Sitka, Alaska, earthquake, J. Geomag. Geoelectr., 31, 441-448, 1979. 
Sadovsky, M. A., I. L. Nersesov, S. K. Nigmatullaev, L. A. Latynina, A. A. LukK, A. N. Semenov, I. G. Simbireva, and V. I. Ulomov, The process preceding strong earthquakes in some regions of Middle Asia, Tectonophysics, 14, 295-307, 1972.

Scholz, C. H., L. R. SyKes, and Y. P. AgGARWAL, Earthquake prediction: a physical basis, Science, 181, 803-810, 1973.

YANAGIHARA, K., Secular variation of the electrical conductivity anomaly in the central part of Japan, Mem. Kakioka Mag. Obs., 15, 1-11, 1972.

YANAGIHARA, K. and T. NAGANo, Time change of transfer function in the central Japan anomaly of conductivity with special reference to earthquake occurrences, J. Geomag. Geoelectr., 28, 157$163,1976$.

Yanagihara, K., M. Kawamura, Y. Sano, and T. Kuboki, New standard magnetic observatory system of Kakioka (KASMMER), Geophys. Mag., 36, 217-281, 1973. 